\title{
Estudio geotécnico asociado a procesos de remoción en masa del parque Francisco Zarco (Ocolusen), Morelia (Michoacán, México)
}

\author{
Antonio Pola-Villaseñor ${ }^{1, *}$, Víctor Hugo Garduño-Monroy ${ }^{2}$, \\ Ciro Díaz-Salgado ${ }^{3}$, Víctor Manuel Hernández-Madrigal ${ }^{2}$ \\ ${ }^{1}$ Universidad Nacional Autónoma de México, Instituto de Geología, \\ Posgrado en Ciencias de la Tierra, Ciudad Universitaria, México, D.F. \\ ${ }^{2}$ Universidad Michoacana de San Nicolás de Hidalgo Instituto de Investigaciones Metalúrgicas, \\ Departamento de Geología y Mineralogía, Edif. "U”, Ciudad Universitaria, Morelia, Michoacán. \\ ${ }^{3}$ Servicio Geológico Mexicano, Subdirección de Investigación. \\ *antonio_pola@yahoo.com.mx
}

\begin{abstract}
Resumen
Durante los últimos cuatro, años la zona sureste de la ciudad de Morelia ha sido afectada por diferentes procesos gravitacionales. La inestabilidad del escarpe es condicionada por factores geológico-estructurales y factores climáticos. La inestabilidad a nivel local fue desencadenada por factores antrópicos, a raíz de la construcción de un camino de acceso hacia la parte alta de la ladera, el cual ha modificado considerablemente el relieve. El presente trabajo se inició con el levantamiento topográfico detallado del área de estudio, sobre el cual se identificaron los diferentes procesos gravitacionales y formas del relieve. Los resultados geotécnicos, cartográficos y de inestabilidad resaltan la importancia de establecer modelos geométricos mediante coeficientes de seguridad y desplazamientos admisibles, los cuales serán requisitos fundamentales en los proyectos de ingeniería, relacionados con obras de mitigación, resguardo y planificación del desarrollo urbano del sector sur de la ciudad de Morelia.
\end{abstract}

Palabras clave: Procesos gravitacionales, factores antrópicos, estudios geotécnicos, Morelia, México.

Abstract

During the last four years the southeastern zone of Morelia city, has been affected by different gravitational processes. The scarp instability is conditioned by geologic-structural and climatic factors. The local instability was unchained by antropic factors, as a result of a road access construction at the top of the hillside, which has considerably modified the relief. This research paper was initiated with the topographic map detailed surveying, where the gravitational process and relief shapes were identified. The geotechnical, cartographical and instability results emphasize the importance of establishing geometric models supported by security coefficients and admissible displacements, which will be fundamental requirements to the engineering projects, related to mitigation works, protection, and urban development planning of the south sector of Morelia city.

Key words: Gravitational processes, antropic factors, geotechnical research, Morelia, Mexico. 


\section{Introducción}

En los últimos diez años, gran parte del desarrollo urbano de la ciudad de Morelia se ha realizado en zonas susceptibles a los procesos de remoción en masa. Un caso específico es el área urbana que se está desarrollando al sur de la ciudad, a todo lo largo del escarpe denominado "La Paloma" (Arreygue-Rocha et al., 2003). Éste constituye un desnivel de $200 \mathrm{~m}$ y una longitud de más de $300 \mathrm{~km}$ provocado por una falla normal regional con dirección E-O, que forma parte del sistema activo de fallas MoreliaAcambay (Garduño-Monroy et al., 2001). En el escarpe se han identificado 8 cuerpos inestables con características litológicas y estructurales muy variables, mismos que han sido desencadenados fundamentalmente por procesos climáticos, morfológicos y sísmicos (Arreygue-Rocha et al., 1998). En general, el relieve de la zona se ha modificado por medio de dos procesos principales: (1) deslizamientos de tipo rotacional donde, según datos de monitoreo, algunos de ellos son considerados inactivos pero susceptibles a reactivarse por algún fenómeno sísmico regional de magnitudes tan pequeñas como 4 grados en la escala de Richter (Domínguez-Ortiz, 2003), y (2) caídas de bloques ligadas al fracturamiento de ignimbritas soldadas y al efecto de la gravedad, donde bloques de hasta $10 \mathrm{~m}^{3}$ podrían generar daños en zonas habitacionales (Rodríguez-Huitrón, 2004). Un ejemplo claro de la inestabilidad de la ladera es el deslizamiento de Ocolusen, el cual presenta un escarpe principal y un escarpe secundario inestable que ha provocado la caída de bloques y fragmentos de roca a lo largo de zonas con pendientes entre $0^{\circ}$ y $55^{\circ}$ (Alditurriaga-González, 2002). Fracturas desplazadas, ubicadas en la parte alta del escarpe principal, corroboran la continua actividad del fenómeno, que se considera iniciado por la actividad sísmica (Garduño-Monroy et al., 2001).

Los datos anteriormente citados sugieren que los procesos de inestabilidad a lo largo del escarpe La Paloma no son únicamente históricos, sino que persisten actualmente. El desarrollo de nuevos fenómenos condicionados por factores físicos y geológico-estructurales, aunados al crecimiento urbano hacia este sector, resaltan la importancia de realizar estudios ligados a la estabilización y mitigación de estos procesos.

La zona de estudio se localiza en la parte sur oriental de la ciudad de Morelia, dentro del parque Francisco Zarco, en el sector de Ocolusen (Figura 1). Durante los últimos cuatro años en este lugar se han generado diferentes procesos gravitacionales, los cuales se iniciaron a partir de la construcción de un camino de acceso a un fraccionamiento que sería ubicado en la parte alta del escarpe. La inestabilidad se hizo más crítica con la presencia de lluvias en su comportamiento temporal, con que los principales procesos generados fueron flujos de detritos, caídas de roca en los escarpes más verticales y movimientos translacionales a lo largo de contactos litológicos con características plásticas contrastantes. Un año después del inicio de la inestabilidad, el gobierno del Estado de Michoacán construyó un muro de contención en la parte más crítica del escarpe, con la finalidad de estabilizar la ladera y proteger a la población en esta zona. Sin embargo, estudios de monitoreo realizados a lo largo de la corona del muro demuestran desplazamientos de hasta $50 \mathrm{~cm}$ en un lapso de seis meses en sus tres dimensiones, los cuales han generado fracturas verticales y horizontales en su paramento.

Los estudios geotécnicos realizados en el presente trabajo se enfocaron en los procesos gravitacionales de la zona, con la finalidad de reconocer parámetros base que podrían ser útiles en el diseño de algún plan de mitigación. Los estudios asociados a la inestabilidad han demostrado la vulnerabilidad en la que se encuentra la población en este sector. Por esta razón se hace necesario delimitar las zonas de riesgo para elaborar un mejor plan de desarrollo urbano, que permita disminuir las afectaciones causadas a la población y a su patrimonio en la zona sur de la ciudad de Morelia.

\section{Características morfológicas del área}

Para el análisis morfológico de la ladera en estudio, fue indispensable contar con un plano topográfico a detalle que se presentara las condiciones del terreno más apegadas a las reales, así como las formas del relieve de la superficie en el momento del estudio. El análisis morfológico y la zonificación de las formas del relieve se realizaron sobre un mapa topográfico detallado a escala 1:1000, el cual se elaboró por medio del método GPS denominado "Tiempo real". Este método consiste en situar un punto dentro de la superficie del terreno, donde se colocara un receptor de referencia (antena, receptor GPS, radios), el cual fue corregido en gabinete con respecto a un punto base de coordenadas conocidas. Con dos receptores GPS móviles obtendremos las coordenadas en proyección UTM (Universal Tranversa de Mercator) en toda la superficie, las cuales serán la base para la obtención de las curvas de nivel. El equipo que se utilizó fue un sistema de tres GPS 500 LEICA con precisión centimétrica, uno usado como estación de referencia montado sobre un tripié y dos equipos móviles montados sobre un bastón. Es importante resaltar que, para la obtención satisfactoria de los datos, fue necesario recibir las señales de los satélites con el mínimo de alteración, es decir que el equipo se debió colocar en sitios libres de obstrucciones tales como árboles, edificios y montañas.

Las características morfológicas de la ladera, están directamente asociadas con un sistema de falla principal E-O y planos conjugados con rumbo $\mathrm{N} 87^{\circ} \mathrm{W}$ y $\mathrm{N} 10^{\circ} \mathrm{E}$ (Figura 7), a lo largo de los cuales se han deslizado grandes masas de suelo y roca, que en la Figura 2 se identifican como paleodeslizamientos. Las formas del relieve corresponden a pequeñas cañadas con pendientes fuertes y escarpes abruptos que están en función de la interacción de los agentes erosivos, con las propiedades físicas y mecánicas 


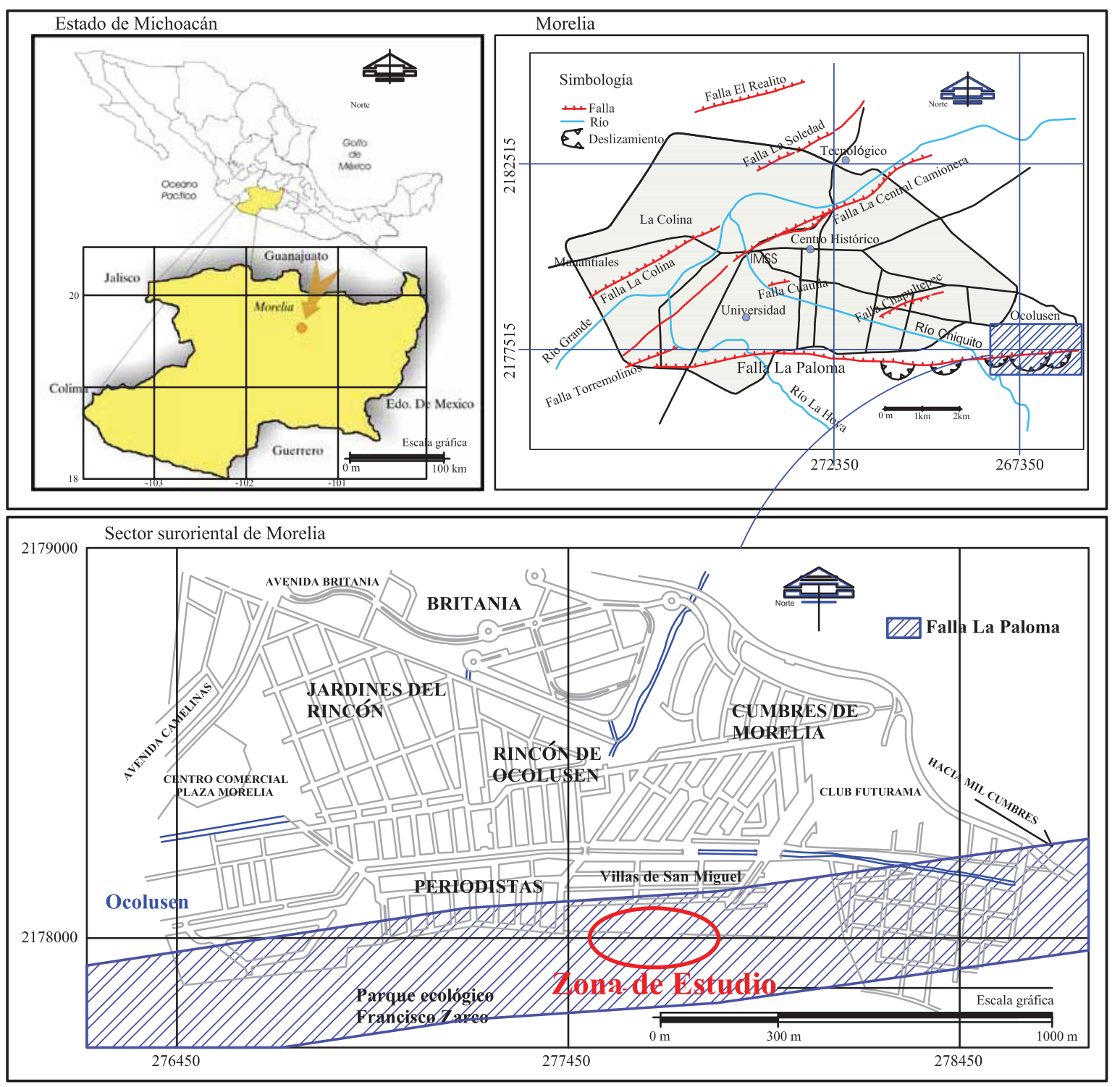

Figura 1. Mapa de localización. Se incluye un croquis del sector sureste de la ciudad de Morelia. El sombreado ubicado en la parte inferior de la figura resalta la influencia de la falla La Paloma.

de la roca. Otro factor que ha interactuado en los cambios de geometría y pendiente, es el camino de acceso hacia la parte alta de la ladera, el cual ha modificado considerablemente el relieve, a razón de generar escarpes muy verticales, terraplenes artificiales y depósitos de material no consolidado, que han cambiado el cauce natural de los escurrimientos de agua.

Uno de los resultados más importantes que se presenta en este trabajo, es la precisión y el detalle con el que se elaboró el mapa topográfico base, con curvas de nivel a cada $50 \mathrm{~cm}$ plasmadas a escala 1:1000 (Figura 2). Con base en la topografía detallada, se realizó un mapa geomorfológico, donde se zonificaron los procesos gravitacionales activos, se delimitaron sus dimensiones y se identificaron las diferentes formas en el relieve, como depósitos de deslizamiento, escarpes principales, coronas, terrazas y fracturas.

\section{Caracterización geológica}

La zona de estudio se localiza en el frente de lo que se ha denominado como Sierra de Mil Cumbres (SMC) en el sector sur de la ciudad de Morelia, a lo largo de un escarpe formado por la falla "La Paloma", la cual es un segmento del sistema regional de fallas Acambay- Morelia. En esta zona afloran secuencias volcánicas del OligocenoMioceno, constituido de lavas, brechas y secuencias de flujos piroclásticos de composición andesítica (Ignimbritas) conocidas como cantera de Morelia (Garduño-Monroy et al., 2002). El relieve de la zona ha sido modificado por obras civiles que han generado pendientes de hasta $85^{\circ}$. Los cortes realizados permitieron observar las diferentes unidades geológicas del sector, las cuales se describen en base al esquema geológico de la Figura 3. 


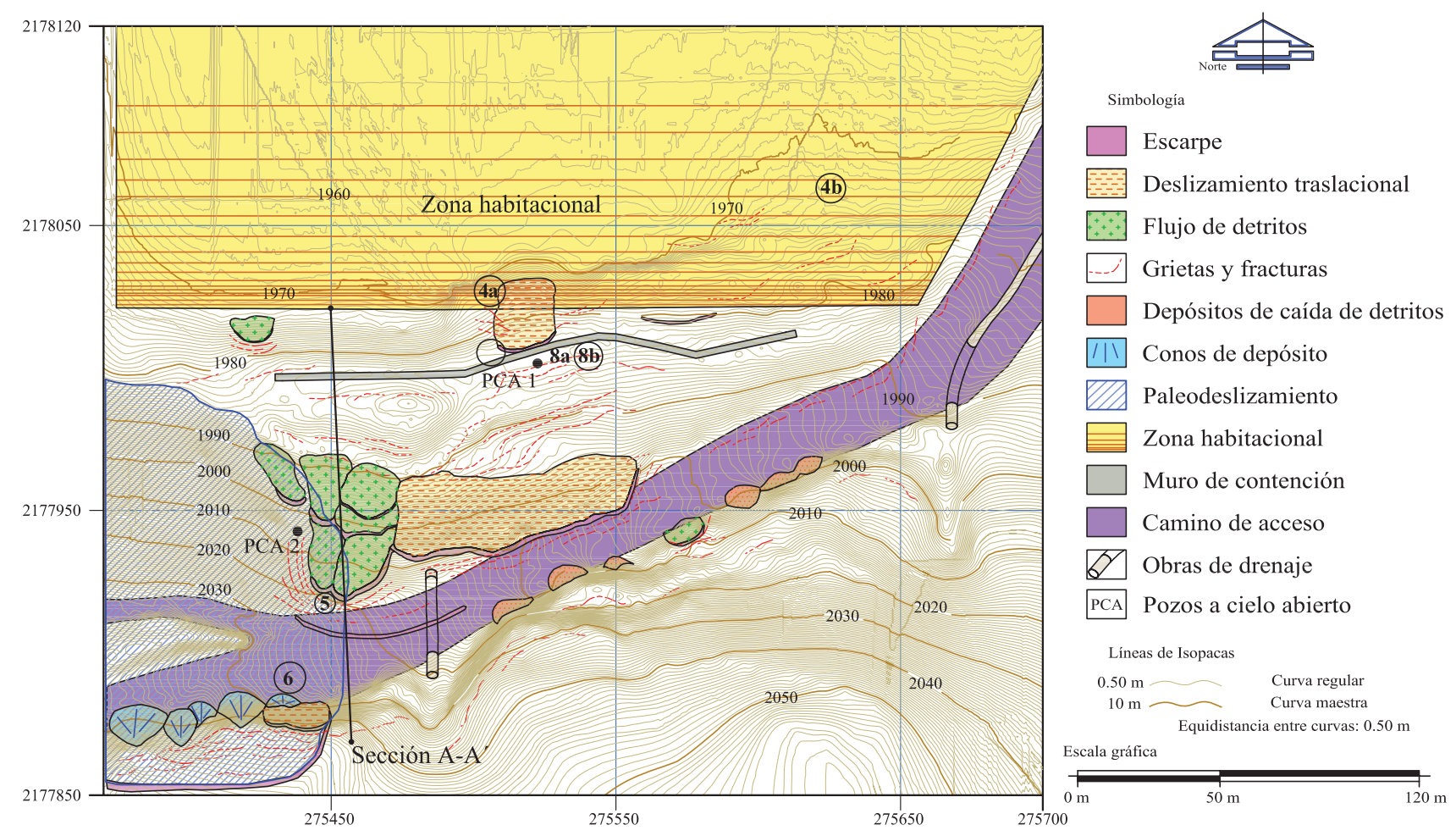

Figura 2. Mapa topográfico-morfológico. Se zonifican los procesos gravitacionales activos y se identifican las diferentes formas en el relieve como: depósitos de deslizamiento, escarpes principales, coronas, terrazas y fracturas, también se ubican los PCA y la sección topográfica A-A' (Figura 7).

La unidad de la base corresponde a una brecha color rosa constituida por bloques de andesita, medianamente cementada y con matriz arenosa (Am). Este paquete se encuentra en la zona suroeste del mapa y forma un relieve de pendientes mayores a $60^{\circ}$, con elevaciones que van desde 1960 hasta 2040 m.s.n.m. Como unidad suprayacente, en la zona sur del mapa aflora una secuencia de lavas y depósitos de avalancha color gris poco consolidada, compuesto de fragmentos angulosos y subangulosos de andesita, con tamaños máximos de $80 \mathrm{~cm} ø$ y fragmentos predominantes de 10 a $20 \mathrm{~cm}$ (Brvm), soportados por una matriz arenosa. Esta unidad sobresale en las paredes verticales generadas por la obra civil que se encuentran en elevaciones que van desde 2000 hasta 2030 m.s.n.m. A su vez, esta secuencia es coronada por depósitos de pómez de caída color amarillo, compuesta por fragmentos de diferentes granulometrías no mayores a $1 \mathrm{~cm} ø$ y fragmentos líticos de composición andesita (Dcap). Esta secuencia presenta un espesor muy variado que va de 0.20 hasta $2 \mathrm{~m}$ hacia la porción oeste del mapa. En la cima de la columna se encuentran depósitos de arcillas color gris oscuro (Ar), asociados a la actividad del talud formado por la falla La Paloma. Este depósito arcilloso, con espesores máximos de $2 \mathrm{~m}$, presenta características plásticas muy marcadas, innumerables planos y estrías de falla en diferentes direcciones. Gran parte de la columna litológica está cubierta por detritos generados por actividad antrópica: excavaciones realizadas para el desplante de un tanque de almacenamiento de agua (Det), depósito de un flujo de detritos no reciente (Cd) y un camino de acceso hacia la parte alta de la ladera (Dc).

El arreglo geométrico de las discontinuidades que corresponden a los planos de falla conjugados afecta a la secuencia de depósitos de avalancha y flujos de lava (Brvm), así como a la unidad de brecha andesítica (Am). Estas discontinuidades juegan un papel importante en cuanto a la inestabilidad de la ladera se refiere, a razón de la erosión y el arrastre de los materiales de relleno debidos a la caída de masas compuestos de material granular y roca.

\section{Propiedades de los suelos}

Las muestras de suelo se recolectaron en pozos a cielo abierto, y los suelos analizados se clasifican como de tipo alterado. Con el fin de reproducir las condiciones del terreno natural, se efectuaron pruebas de humedad natural y peso volumétrico del lugar. El suelo se clasificó de manera global en base a la carta de plasticidad de Casagrande, en conjunto con la carta propuesta por el Sistema Unificado de Clasificación de Suelos (SUCS) (Braja, 1999). Pruebas adicionales, como grado de compresibilidad, y grado de saturación y porosidad (resultado muy ligado a la deformación que podría sufrir el suelo, bajo esfuerzos significativos), fueron elaboradas con la finalidad de atribuir características físicas y plásticas muy particulares. Los resultados mecánicos de los suelos se obtuvieron con base 


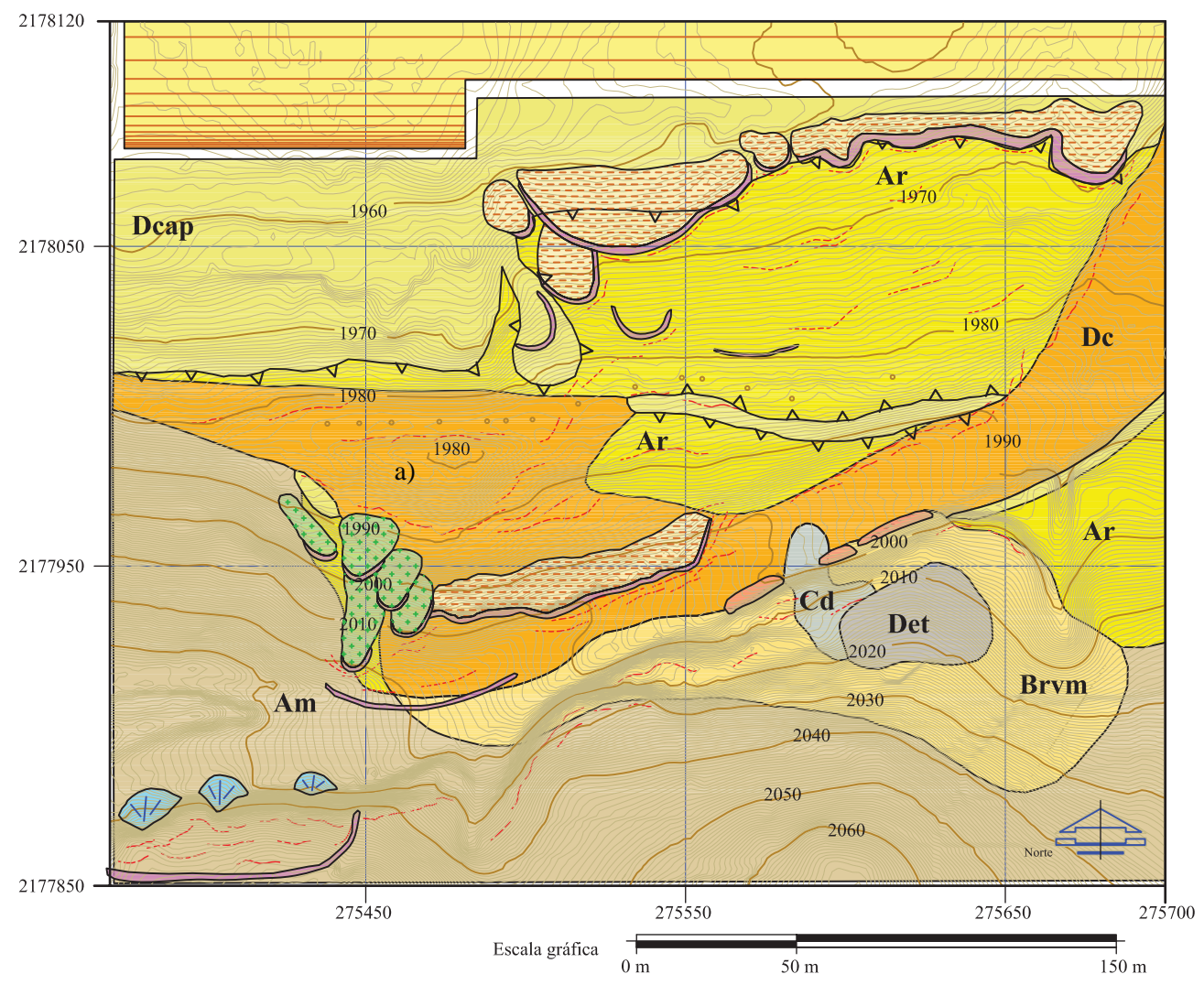

Simbología y columna litológica

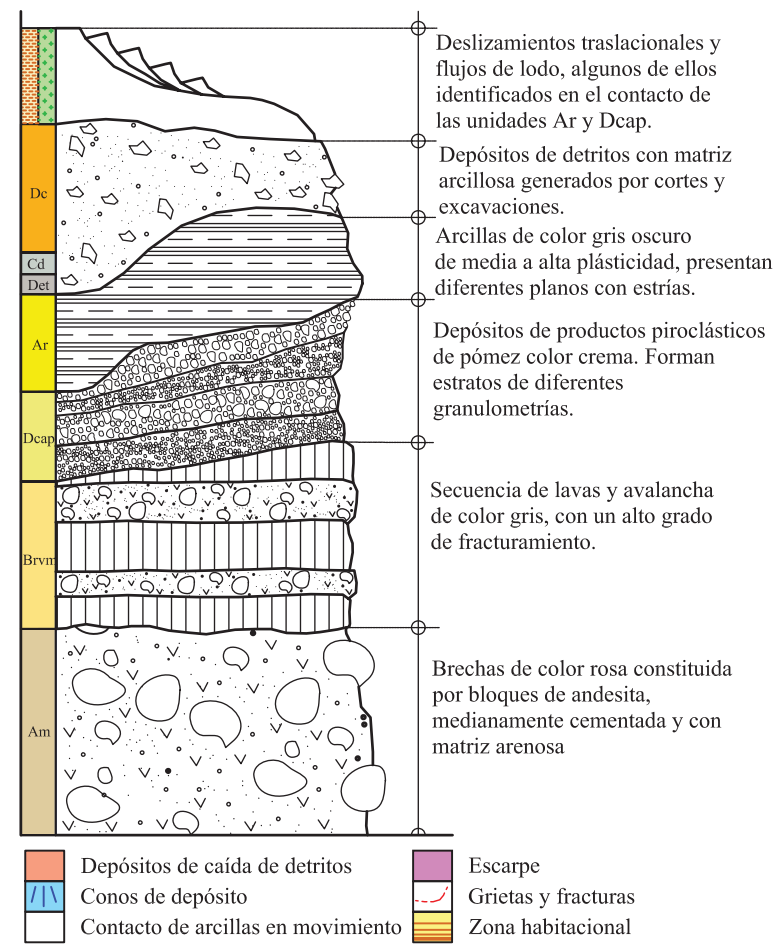

NOTA: La columna geológica representantada en esta figura, no se elaboro a ninguna escala gráfica.

Figura 3. Mapa geológico y columna litológica de la zona de estudio. Se aprecia la sobreposición de unidades con características físicas muy diferentes. 
en pruebas de compresión simple y compresión triaxial. En el ensayo de compresión triaxial, se intentó reproducir las condiciones reales a las que el suelo está sometido, aplicando así presiones de confinamiento semejantes a las existentes en campo. Las propiedades de los suelos muestreados obtenidas en el laboratorio se representan en la Tabla 1 y se describen a continuación:

PCA 1: esta muestra proviene de materiales producto del corte (unidad Dc), a sólo algunos metros de los flujos de detritos (Figura 2). En el afloramiento, se excavó hasta una profundidad de $1.20 \mathrm{~m}$, donde se recolectó una mezcla de arena, fragmentos de roca de diversos tamaños, soportados por una matriz arcillosa de color café. Su clasificación ingenieril corresponde a Arcillas inorgánicas $(\mathrm{CH})$ de alta plasticidad muy impermeables, con alto grado de susceptibilidad a la contracción y expansión al variar el contenido de humedad. Con base en los valores de consistencia relativa y valores de compresión simple, la muestra se clasifica como de tipo rígida, con características apreciables de resistencia al corte. Como el valor de la contracción lineal del suelo es mayor a $9 \%$, se dice que el suelo tendrá una actividad significativa de contracción-expansión. La relación de vacíos (e) y porosidad (n) indica características de suelos susceptibles a deformación. Finalmente con los valores de índice de liquidez, podemos clasificar a la muestra como de tipo preconsolidado (Crespo-Villalaz,

Tabla 1. Características físicas y mecánicas de las muestras de suelo. Con base en resultados como límites de consistencia y grado de saturación, se atribuyeron características plásticas muy particulares.

\begin{tabular}{|c|c|c|}
\hline No. de pozo a cielo abierto & 1 & 2 \\
\hline Profundidad del PCA ( m ) & 1.2 & 0.6 \\
\hline Descripción & Arcilla café & Arcilla negra \\
\hline \multicolumn{3}{|l|}{ Granulometría } \\
\hline$\%$ que pasa la malla no. $4(4.75 \mathrm{~mm})$ & 96.15 & 79.08 \\
\hline$\%$ que pasa la malla no. $40(0.42 \mathrm{~mm})$ & 90.94 & 74.12 \\
\hline$\%$ que pasa la malla no. $200(0.075 \mathrm{~mm})$ & 83.12 & 64.91 \\
\hline \multicolumn{3}{|l|}{ Límites de Consistencia } \\
\hline Límite líquido \% (Ll) & 63.6 & 54.3 \\
\hline Límite plástico \% (Lp) & 30.0 & 32.3 \\
\hline Índice plástico \% (Ip) & 33.6 & 22.0 \\
\hline Contracción lineal \% $(\mathrm{Cl})$ & 18.9 & 13.9 \\
\hline Límite de Contracción \% (Lc) & 7.04 & 19.0 \\
\hline Consistencia relativa $(\mathrm{Cr})$ & 0.817 & 0.70 \\
\hline Índice de liquides & 0.183 & 0.29 \\
\hline Clasificación S.U.C.S & $\mathrm{CH}$ & $\mathrm{OH}$ \\
\hline \multicolumn{3}{|l|}{ Pruebas de resistencia } \\
\hline Compresión simple $\left(\mathrm{kg} / \mathrm{cm}^{2}\right)$ & 1.724 & 1.670 \\
\hline Ángulo de fricción interna $\left({ }^{\circ}\right)$ & 17 & 18 \\
\hline Cohesión $\left(\mathrm{kg} / \mathrm{cm}^{2}\right)(\mathrm{c})$ & 0.5 & 0.75 \\
\hline Módulo de elasticidad (kg/cm²) (Ec) & 115.68 & 178.96 \\
\hline \multicolumn{3}{|l|}{ Relaciones Gravimétricas } \\
\hline Peso volumétrico del lugar $\left(\mathrm{kg} / \mathrm{m}^{3}\right)$ & 1754 & 1700 \\
\hline Densidad de sólidos (Ss) & 2.66 & 2.37 \\
\hline Relación de vacíos (e) & 1.034 & 0.93 \\
\hline Porosidad \% (n) & 50.85 & 48.27 \\
\hline Grado de saturación \% (Gw) & 93 & 98 \\
\hline
\end{tabular}

1980), lo que quiere decir que el suelo ha estado sometido a esfuerzos producidos por excavaciones del terreno. El grado de saturación $(\mathrm{GW}=93 \%)$ tan alto que presenta la muestra indica la vulnerabilidad a deformaciones y movimientos bajo esfuerzos generados por el contenido de agua.

PCA 2: esta muestra proviene de materiales arcillosos (unidad Ar), a sólo algunos metros del desplante del muro de contención (Figura 2). En el afloramiento, se excavó hasta una profundidad de $0.60 \mathrm{~m}$, donde se recolectó una mezcla de arcilla negra. Su clasificación ingenieril corresponde a Arcillas orgánicas $(\mathrm{OH})$ de media a alta plasticidad impermeables, las cuales en estado no saturado pueden presentar movimientos bruscos y bajo condiciones de carga e incremento de humedad. Con base en los valores de consistencia relativa y valores de compresión simple, la muestra se clasifica como de tipo rígida, con características apreciables de resistencia al corte. Como el valor de la contracción lineal del suelo es mayor a $9 \%$, se dice que el suelo tendrá una actividad significativa de contracción-expansión. La relación de vacíos (e) y porosidad (n) indica características de suelos susceptibles a deformación. Finalmente, con los valores de índice de liquidez, podemos clasificar a la muestra como de tipo preconsolidado (Crespo-Villalaz, 1980), lo que quiere decir que el suelo ha estado sometido a esfuerzos producidos por movimientos del terreno. El grado de saturación $(\mathrm{GW}=98 \%)$ tan alto que presenta la muestra, indica la vulnerabilidad a deformaciones y movimientos bajo esfuerzos generados por el contenido de agua.

\section{Tipos de movimiento}

Los fenómenos de inestabilidad en el parque Francisco Zarco están condicionados por tres factores principales: (1) la formación geológica de la región, a razón de la sobreposición de unidades con características físicas y mecánicas muy diferentes, (2) la geometría de las estructuras que son generadas por la actividad de la falla La Paloma, y (3) la percolación y escurrimientos de agua que incrementan la presión de poro en materiales arcillosos, donde la evidencia más notable son las características físicas derivadas de laboratorio (Tabla 1). Como factor desencadenante, tenemos los cortes realizados para la construcción de la vía de acceso hacia la parte alta del escarpe, donde los depósitos no consolidados están jugando un papel muy importante en cuanto a inestabilidad se refiere. Como resultado de la interacción de estos factores aunados a las características del relieve, tenemos en la zona tres tipos de fenómenos de remoción en masa: (1) deslizamientos traslacionales, (2) flujos de detritos, y (3) caídas de roca.

\subsection{Deslizamientos traslacionales}

En la parte central del área cartografiada (Figura 2) se 
identifican deslizamientos traslacionales que involucran el movimiento de materiales arcillosos, los cuales han sido depositados gracias a la actividad del talud. Este tipo de fenómeno tiene lugar en pendientes no mayores a $20^{\circ}$, donde el plano o superficie de movimiento corresponde al contacto litológico entre los depósitos de arcilla (Ar) y los depósitos de pómez (Dcap), que son unidades litológicas con características físicas muy diferentes. La trayectoria de la masa arcillosa genera grietas perpendiculares a su movimiento, las cuales experimentan separaciones y hundimientos, que finalmente se convierten en escalones bien definidos con desniveles de hasta $1.50 \mathrm{~m}$ (Figura 4a y 4b).

\subsection{Flujos de detritos y material granular}

Este proceso corresponde al movimiento más activo de la zona, el cual involucra tanto materiales producto de los cortes antrópicos (Dc), como materiales arcillosos (Ar) y cubierta vegetal. Los flujos se localizan en la zona suroeste de la zona cartografiada (Figura 5), en taludes artificiales con pendientes entre $50^{\circ}$ y $60^{\circ}$. La masa total desplazada bajo condiciones de saturación ha sido el producto de movimientos sucesivos, donde su distribución ha generado fisuras sub-perpendiculares de hasta $8 \mathrm{~m}$ de largo y $40 \mathrm{~cm}$ de separación.

\subsection{Caídas y volcamientos de roca}

Este tipo de proceso se presenta a lo largo de escarpes antrópicos muy inclinados $\left(75^{\circ}\right)$, en dos unidades principalmente: una unidad compuesta por bloques de andesita (Am) medianamente cementada y una secuencia de lavas y avalanchas color gris (Brvm) muy fracturadas. Las superficies que generan la inestabilidad corresponden a las diferentes familias de discontinuidades que se orientan adversamente a la cara del talud y que están siendo rellenadas por materiales arcillosos que, a su vez, son arrastrados por factores climáticos, para provocar la caída de grandes bloques de hasta $80 \mathrm{~cm}$ de diámetro ().

\section{Análisis de estabilidad y factor de seguridad por falla circular}

El análisis de estabilidad se realizó con la finalidad de ubicar los diferentes planos de falla crítica a lo largo del escarpe. En el análisis se utilizó el programa XSTABL (Slope Stability Analysis), el cual busca y analiza 100 superficies críticas de deslizamiento a lo largo de planos circulares (Sharma, 1991). En la búsqueda de los planos de falla se seleccionó el método simplificado de Bishop (1955), el cual separa la masa deslizante por medio de una superficie de falla circular. La masa deslizante se dividirá a su vez en "n" rebanadas o fajas verticales, estableciendo así
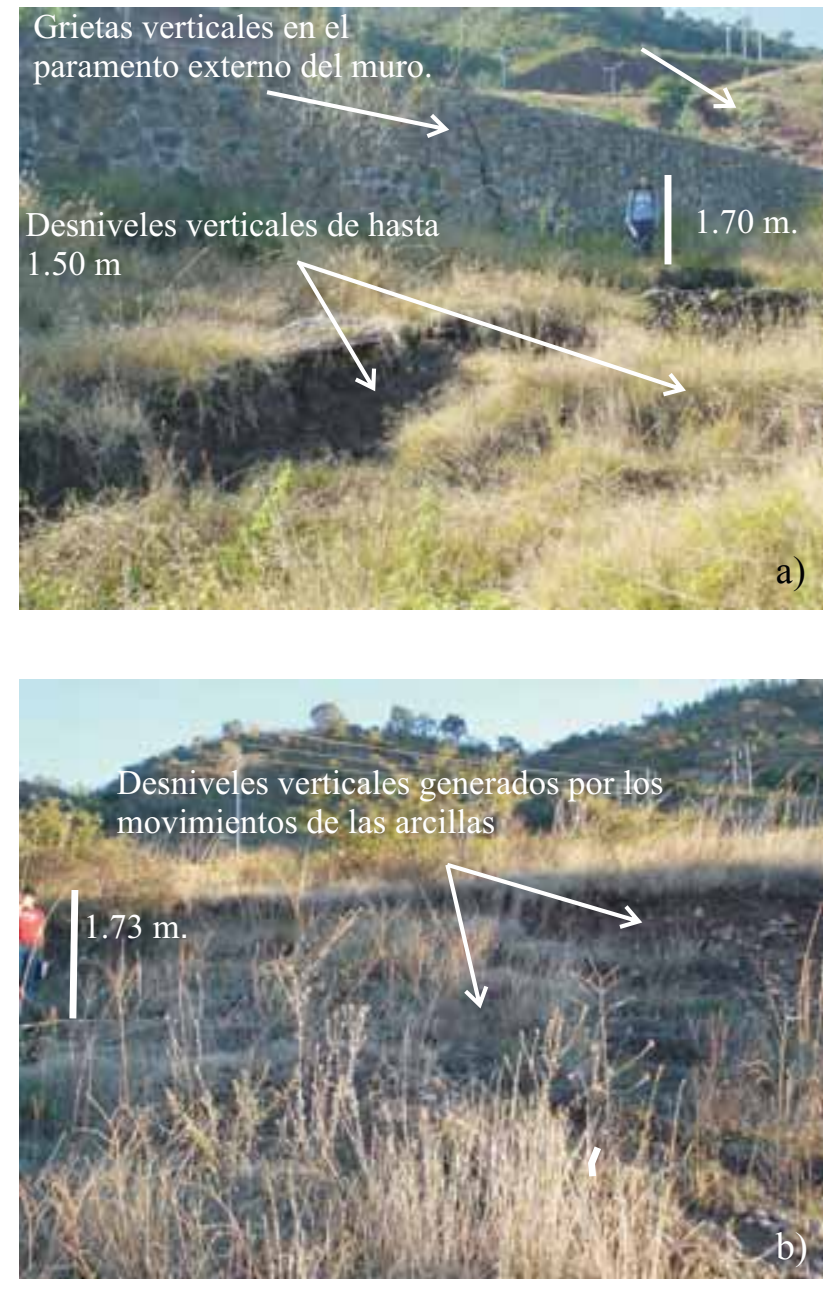

Figura 4. a) Deslizamiento translacional, a lo largo del contacto de las unidades de arcilla (Ar) y depósitos de pómez (Dcap). Se puede apreciar los grandes desniveles que han sido generados por el desplazamiento de la masa (Septiembre 2004). b) Deslizamiento translacional, en pendientes de $10^{\circ}$. La ubicación del deslizamiento, ahora corresponde a un conjunto habitacional (Enero 2003).

el equilibrio de las fuerzas actuantes en cada rebanada con respecto al centro de la curva o plano de falla (González de Vallejo et al., 2002). Para realizar la modelación, fue necesario elaborar un perfil o sección topográfica que incluya datos litológicos, parámetros geométricos y propiedades mecánicas de los materiales involucrados (Tabla 1). Como resultado de la modelación se obtuvieron 10 planos de falla graficados dentro del área que se delimitó, con base en observaciones de campo y a trabajos de gabinete (Figura 7). La sección topográfica o perfil se trazó a lo largo de una línea de $150 \mathrm{~m}$ de longitud, con coordenadas 275457.04 este, 2177868.7 norte, y 275449.75 este, 2178021.0 norte, a lo largo de la que se identifico como una superficie inestable crítica (Figura 2). Las unidades geológicas se ubicaron con base en el mapa geológico y columna litológica de la zona (Figura 3), así como en base a estudios geofísicos de sondeos eléctricos verticales. El factor de seguridad (relación de fuerzas resistentes y fuerzas motores) que 


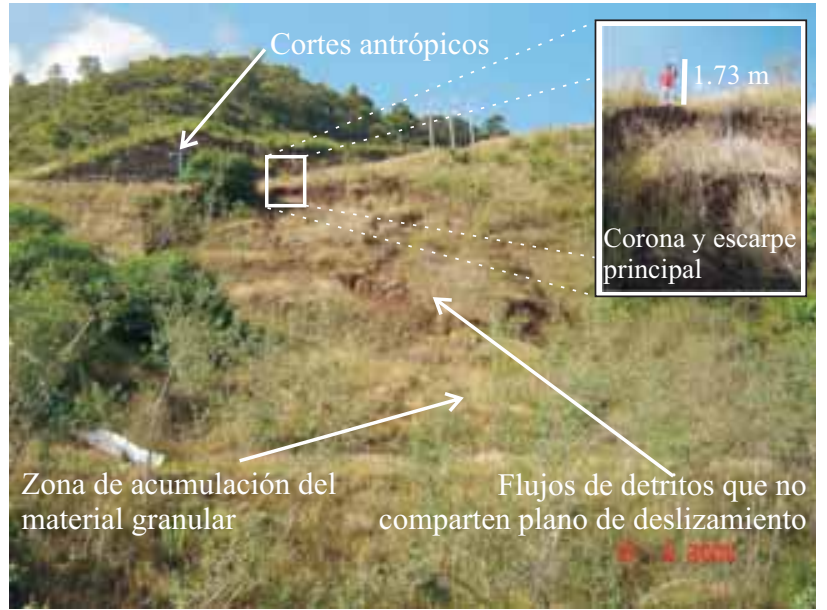

Figura 5. Flujo de detritos y material granular, el cual toma lugar en unidades de arcillas (Ar) y detritos generados por las obras civiles (Dc). Se identifica la corona de deslizamiento y el escarpe principal, así como los cortes realizados por la obra civil (Septiembre 2004).

condiciona el plano de falla crítica con valor de 0.66 , fue generado bajo condiciones húmedas, intentando reproducir las condiciones reales a las que la ladera está sometida. Uno de los aspectos no considerados para la modelación, fue el factor sísmico, cabe mencionar que este aspecto puede ser sin duda un factor adicional en la estabilidad de la ladera, a razón de que la zona de falla donde se ubica el estudio, ha sido considerada como potencialmente sísmica (GarduñoMonroy et al., 2001). Finalmente, con base en los resultados de la modelación se elaboró una sección geológica (Figura 7) en que se ubican los posibles planos de deslizamiento que delimitan la masa inestable. Ésta genera deformaciones, movimientos, asentamientos y fracturas tanto en el camino de acceso como en el muro de contención y obras de drenaje ubicadas en cotas inferiores.

\section{Factores de seguridad del muro de contención}

El desarrollo de los diferentes procesos de remoción en masa en la zona sur oriental del escarpe está condicionado por factores antrópicos aunados a factores físicos y geológicos. El crecimiento urbano hacia este sector resalta la importancia de realizar proyectos relacionados con obras de mitigación, avalados por estudios geológicos y geotécnicos detallados. Como ya se mencionó anteriormente, el gobierno del Estado de Michoacán construyó un muro de contención perpendicular a la sección que en este trabajo se identifica como la más crítica (sección A-A') (Figura 2). El muro de contención se construyó con la finalidad de estabilizar la ladera y resguardar la seguridad de la población en esta zona. Sin embargo, los resultados obtenidos en este trabajo ponen en duda el buen funcionamiento del mismo.

Con la finalidad de estimar el posible volumen inestable generado por los cortes carreteros, Garduño-Monroy et al.

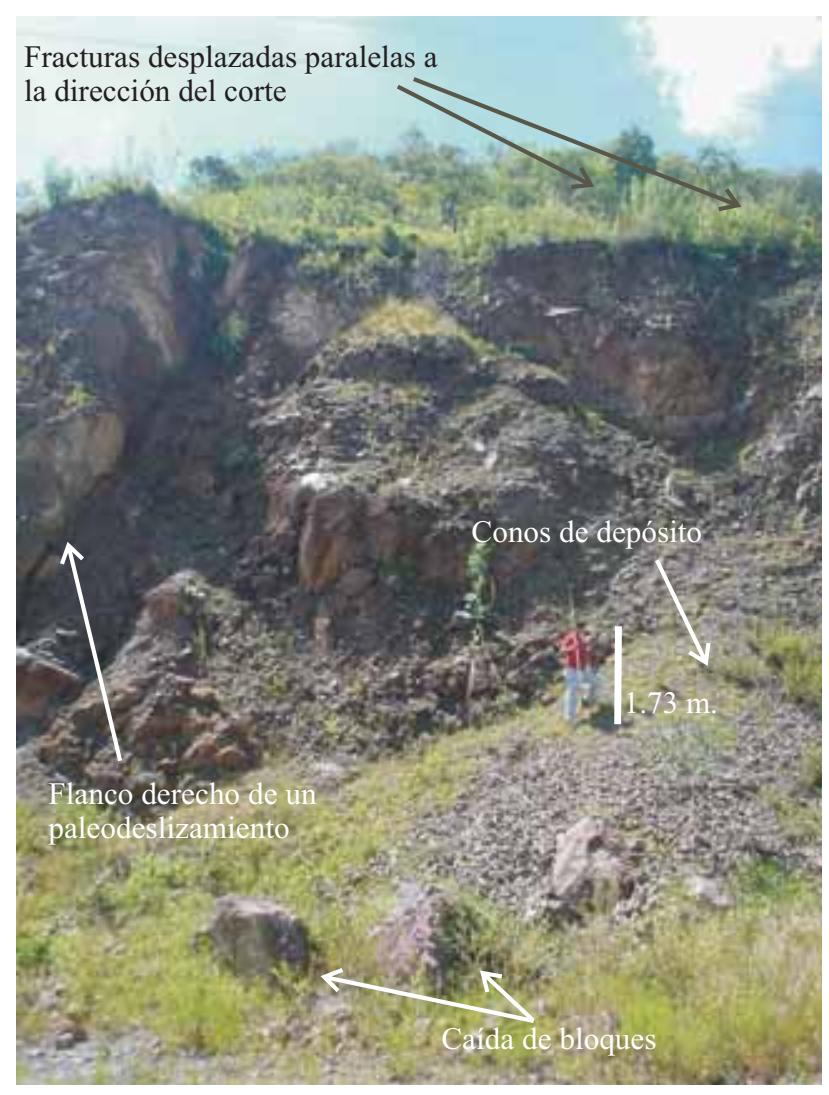

Figura 6. Vuelcos, desplomes y depósitos de caída acumulados en la parte baja de los cortes realizados por la obra civil. La discontinuidad corresponde al plano de movimiento de un paleodeslizamiento (Septiembre 2004).

(2002) definieron un área en donde los productos generados por los diferentes procesos de inestabilidad pueden ser soportados por el muro de contención. Como primer cálculo se realizó una comparación entre la superficie actual del terreno (Figura 2) con la superficie original que predominaba antes de iniciarse el camino de acceso. De esta manera, se obtuvo un volumen inestable conformado por $34,853 \mathrm{~m}^{3}$ de detritos y bloques de roca. Finalmente, a la superficie actual del terreno se le restó el volumen capaz de almacenar el muro de contención. Como resultado, se obtuvo un volumen de $7,278 \mathrm{~m}^{3}$. Por consiguiente, los resultados muestran que el muro de contención no tiene la capacidad de retener todo el volumen posiblemente deslizado. Cabe mencionar que esta estimación se realizó considerando el buen funcionamiento de la obra civil.

Datos geométricos del muro de contención como altura (H), ancho (B) y profundidad de desplante (z), en conjunto con datos geotécnicos como cohesión (c) y ángulo de fricción interna $(\phi)$, derivados de pruebas de compresión triaxial, fueron necesarios para la revisión de la susceptibilidad que tendría el muro a voltearse, deslizarse o hundirse. En busca del factor de seguridad al deslizamiento, fue necesario calcular la presión lateral de tierras, para lo cual se utilizó el método de Rankie (Braja, 1999). El factor de seguridad al volteo se obtuvo con base en la sumatoria 
Superficies de deslizamiento críticas

(Factor de seguridad minimo 0.660, sobre el plano crítico máximo)

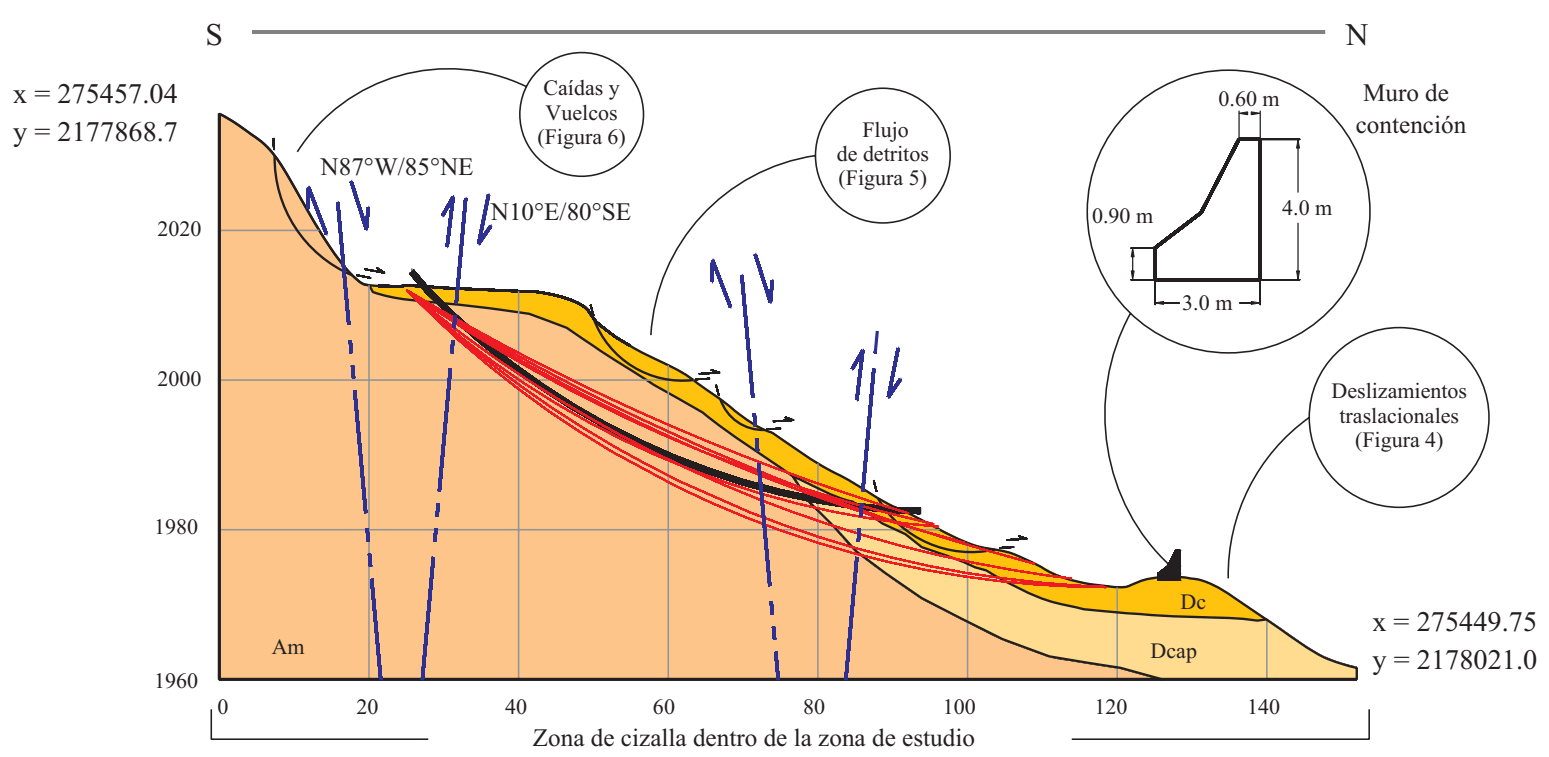

Figura 7. Perfil geológico de la zona (Sección A-A' de la Figura 2), se incluyen fracturas asociadas a la actividad de la falla, diferentes planos teóricos de deslizamiento a lo largo del talud, así como fenómenos de inestabilidad y localización del muro de contención.

de momentos generados por el peso del muro y la masa de suelo, con respecto a un punto situado en uno de los extremos de la cementación. Finalmente, en busca del factor de seguridad al hundimiento, fue necesario conocer la capacidad de carga del suelo, para lo cual se utilizó la fórmula de Terzaghi (Braja, 1999) para una cementación corrida (Tabla 2). El resultado numérico del factor de seguridad al deslizamiento reconoce la susceptibilidad del muro de contención a presentar desplazamientos que podrían generar fracturas y deformaciones en su paramento. Un ejemplo claro de ello son las fracturas verticales y horizontales que el muro presenta (Figura 8a, 8b.), así como los daños sufridos en las cunetas que se encuentran al pie de la obra. Éstos ocasionan infiltraciones que generan presiones hidrostáticas en el plano de desplante, correspondiente en gran parte a una superficie de contacto entre los depósitos

Tabla 2. Resumen del análisis de los factores de seguridad del muro de contención. Se incluyen parámetros de revisión, fórmulas y descripciones de los resultados.

\begin{tabular}{|c|c|c|c|}
\hline Parámetros de revisión & Fórmulas & Factores de seguridad & Resultados \\
\hline $\begin{array}{l}\text { Dimensiones del muro } \\
\mathrm{H}=\text { Altura del muro }(4 \mathrm{~m})\end{array}$ & $\mathrm{z}$ & Deslizamiento. & $\begin{array}{l}\mathbf{1 . 5}<\text { F.s. }{ }_{\text {D }} \\
\text { En base a la comparación }\end{array}$ \\
\hline $\begin{array}{l}Z=\text { Profundidad de desplante }(0.60 \\
\mathrm{m})\end{array}$ & $\begin{array}{l}\text { Empuje de tierras } \\
\text { (Rankie) }\end{array}$ & $\begin{array}{l}\text { F.S. }=\text { Fuerzas verticales } \\
\text { (cd) }\end{array}$ & $\begin{array}{l}\text { del resultado obtenido con el factor de seguridad } \\
\text { ya establecido }(1.5>1.2) \text {. }\end{array}$ \\
\hline $\mathrm{B}=\operatorname{Ancho}(3 \mathrm{~m})$ & $\mathrm{Ea}=\gamma(\underline{\mathrm{H}})^{2}(1-\operatorname{sen} \phi)$ & $\begin{array}{l}\text { Fuerzas horizontales } \\
\mathbf{1 . 5}<\text { F.s. }\end{array}$ & $\begin{array}{l}\text { el muro estará sometido a las deformaciones suf- } \\
\text { ridas por posibles problemas de deslizamiento. }\end{array}$ \\
\hline Parámetros del suelo & $(1+\operatorname{sen} \phi)$ & & \\
\hline$\gamma=$ Peso volumétrico $\left(1754 \mathrm{~kg} / \mathrm{m}^{3}\right)$ & & Volteo. & $\mathbf{1 . 5}<$ F.s. $_{\mathrm{v}}$ \\
\hline $\begin{array}{l}\varphi=\text { Ángulo de fricción }\left(17^{\circ}\right) \\
\mathrm{c}=\text { Cohesión }\left(5000 \mathrm{~kg} / \mathrm{m}^{2}\right)\end{array}$ & $\left.\mathrm{Ep}=\gamma_{2}(\underline{\mathrm{H}})^{2} \operatorname{tg}^{2} \underset{4}{(\pi}-\underset{2}{-\phi}\right)$ & F.S. $=\frac{\sum \text { de momentos }}{\mathrm{Ea}(\mathrm{H} / 3)}$ & $\begin{array}{l}\text { Como resultado de la fórmula obtenido (3.76) es } \\
\text { mayor que el F.S. establecido, el muro no tendrá }\end{array}$ \\
\hline $\begin{array}{l}\mathrm{Ea}=\text { Empuje activo } \\
\mathrm{Ep}=\text { Empuje pasivo }\end{array}$ & Capacidad de carga & $1.5<$ F.s. $_{v_{v}}$ & problemas de volcamiento. \\
\hline $\begin{array}{l}\mathrm{Cd}=\text { Coeficiente de deslizamiento } \\
\text { (Depende del suelo en donde fue } \\
\text { desplantado el muro) } \\
\mathrm{qd}=\text { Capacidad de carga }\end{array}$ & $\begin{array}{l}\mathrm{qd}=\mathrm{c}(\mathrm{Nc})+\gamma(\mathrm{z})(\mathrm{Nq})+0.5(\gamma)(\mathrm{B})(\mathrm{Nw}) \\
(\mathrm{Nc}, \mathrm{Nq}, \mathrm{Nw}) \text { Factores de carga }\end{array}$ & $\begin{array}{l}\text { Hundimiento } \\
\text { qd/3 }>\text { Peso que ejerce } \\
\text { el muro }\end{array}$ & $\begin{array}{l}\text { qd > Peso que ejerce el muro } \\
\text { Como qd es mayor que el peso ejercido. El muro } \\
\text { no tendrá problemas de hundimiento. }\end{array}$ \\
\hline
\end{tabular}




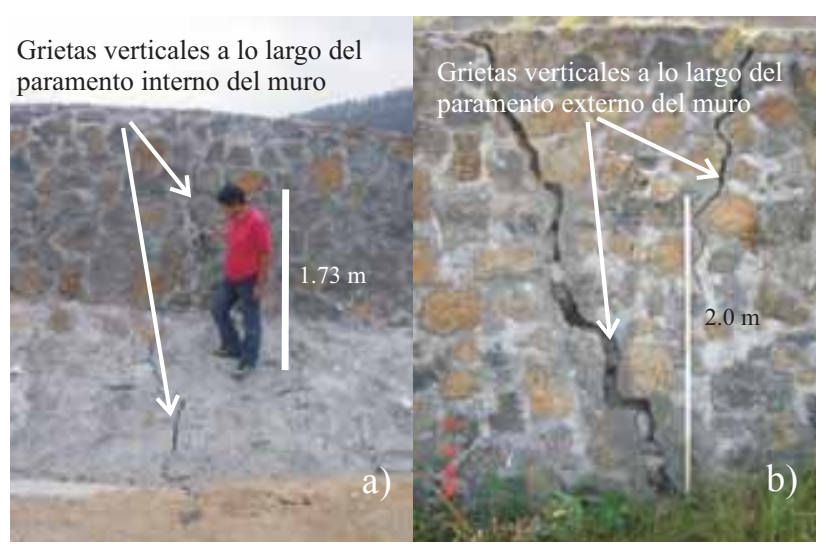

Figura 8a, 8b. Grietas verticales, generadas por el movimiento del muro de contención. La diferencia de tiempo en la captura de las fotos muestra el ensanchamiento progresivo de las grietas verticales. (Enero 2003, Septiembre 2004).

de arcilla (Ar) y los depósitos de pómez (Dcap) (Figura 7). La evolución de los desplazamientos sufridos por el muro de contención se reconoció con base a trabajos de monitoreo realizados en un lapso de seis meses (Agosto 28, 2002 a Enero 31, 2003). Dicho monitoreo consistió en colocar 14 puntos fijos a lo largo de su corona, para posteriormente obtener la diferencia de sus coordenadas geográficas en sus tres dimensiones, las cuales llegaron a ser hasta de $50 \mathrm{~cm}$ (Tabla 3 ).

\section{Conclusiones}

La inestabilidad del escarpe de falla "La Paloma" está ligada a las condiciones geológico-estructurales y climáticas de la región. El complejo global corresponde a la actividad del talud y la sobreposición de unidades con características mecánicas contrastantes. La percolación y escurrimientos de agua juegan un papel muy importante en el control de este tipo de fenómenos, a razón de las propiedades físicas que presentan los materiales involucrados (grado de retención de agua, susceptibilidad a deformaciones y variaciones volumétricas apreciables).

Aunado a las condiciones anteriormente mencionadas, podemos citar a los factores antrópicos, que corresponden a la construcción de un camino de acceso hacia la parte alta de la ladera, la cual ha desencadenado tres tipos de movimientos principales: a) deslizamientos translacionales, generados por la sobreposición de terraplenes artificiales (Dc) y depósitos de arcilla (Ar), b) flujos de detritos activos, originados por la saturación de los materiales producto de los cortes antrópicos (Dc), c) caídas y volcamientos de roca, condicionados en gran parte por la geometría de las discontinuidades en taludes de gran buzamiento.

Los resultados numéricos de los factores de seguridad, el monitoreo de desplazamientos en sus tres direcciones y el modelo de una superficie teórica de falla circular, realizados sobre el muro de contención, indican que su diseño es deficiente.

La metodología empleada en la elaboración del plano topográfico base resultó ser muy práctica y confiable. Los resultados más atractivos derivan del detalle y la escala $(1: 1000)$ en el que el plano es representado. De esta manera, el levantamiento topográfico tan detallado permitió identificar en gabinete los diferentes rasgos estructurales y formas del relieve, así como evaluar de manera más realista los efectos de un movimiento en masa en un terreno potencialmente inestable.

El conjunto de los resultados anteriormente descritos resalta la importancia de establecer modelos geológicos, geotécnicos y geométricos de la inestabilidad, mediante coeficientes de seguridad y desplazamientos admisibles, los cuales serán requisitos fundamentales en los proyectos de ingeniería relacionados con obras de mitigación, resguardo y planificación del desarrollo urbano del sector sur de la ciudad de Morelia.

Tabla 3. Coordenadas de monitoreo en proyección UTM. La resta de las dos mediciones representa los desplazamientos sufridos por el muro de contención en un periodo de seis meses.

\begin{tabular}{cccccccccc}
\hline ID & $\mathbf{x}$ & $\mathbf{y}$ & $\mathbf{z}$ & $\mathbf{x}$ & $\mathbf{y}$ & $\mathbf{z}$ & $\Delta \mathbf{x}$ & $\Delta \mathbf{y}$ & $\Delta \mathbf{z}$ \\
\hline 1 & 275437.99 & 2177994.497 & 1984.482 & 275437.987 & 2177994.516 & 1984.459 & 0.002 & 0.019 & -0.023 \\
2 & 275447.98 & 2177994.671 & 1984.954 & 275447.956 & 2177994.684 & 1984.436 & 0.019 & 0.013 & -0.518 \\
3 & 275458.06 & 2177994.876 & 1984.521 & 275458.040 & 2177994.895 & 1984.497 & 0.016 & 0.019 & -0.024 \\
4 & 275473.03 & 2177995.137 & 1984.575 & 275473.006 & 2177995.164 & 1984.546 & 0.027 & 0.027 & -0.029 \\
5 & 275487.98 & 2177995.311 & 1984.704 & 275487.953 & 2177995.338 & 1984.680 & 0.026 & 0.027 & -0.024 \\
6 & 275493.88 & 2177995.458 & 1987.129 & 275493.847 & 2177995.490 & 1987.110 & 0.033 & 0.032 & -0.019 \\
7 & 275502.18 & 2177995.757 & 1986.817 & 275502.127 & 2177995.845 & 1986.796 & 0.052 & 0.088 & -0.021 \\
8 & 275516.59 & 2178000.199 & 1986.239 & 275516.400 & 2178000.780 & 1986.207 & 0.194 & 0.581 & -0.032 \\
9 & 275534.32 & 2178005.543 & 1985.675 & 275534.218 & 2178006.067 & 1985.614 & 0.101 & 0.524 & -0.061 \\
10 & 275548.86 & 2178008.826 & 1984.796 & 275548.843 & 2178008.928 & 1984.779 & 0.013 & 0.102 & -0.017 \\
11 & 275555.22 & 2178008.532 & 1984.923 & 275555.197 & 2178008.599 & 1984.899 & 0.019 & 0.067 & -0.024 \\
12 & 275569.35 & 2178005.648 & 1985.899 & 275569.333 & 2178005.693 & 1985.880 & 0.018 & 0.045 & -0.019 \\
13 & 275583.27 & 2178002.363 & 1987.189 & 275583.251 & 2178002.401 & 1987.168 & 0.023 & 0.038 & -0.021 \\
14 & 275614.75 & 2178009.097 & 1985.776 & 275614.738 & 2178009.162 & 1985.752 & 0.012 & 0.065 & -0.024 \\
\hline
\end{tabular}




\section{Agradecimientos}

Este trabajo se llevó a cabo durante los estudios de maestría del primer autor. Los autores agradecen el apoyo otorgado por CONACYT. Un agradecimiento muy especial a la Dra. Aline Concha Dimas por su caluroso y gentil trato, el apoyo proporcionado y sus invaluables sugerencias. Se agradece a los doctores Lucia Capra, Armando García Palomo y José Juan Zamorano Orozco, cuyas sugerencias enriquecieron el escrito.

\section{Referencias bibiliográficas}

Alditurriaga-González, O., 2002, Caracterización de los materiales involucrados en el deslizamiento en masa del Campestre en la ciudad de Morelia, Michoacán: Morelia, Universidad Michoacana de San Nicolás de Hidalgo, Facultad de Ingeniería Civil, Tesis de licenciatura, $134 \mathrm{p}$.

Arreygue-Rocha, E., Canuti, P., Iotti, A., Garduño-Monrroy., V.H., 1998 Hydrogeological hazard in the urban area of Morelia, Mich. (México), in Association for Engineering Geology and the Environment, Eight International Congress International, proceedings, Vancouver, Canada: Rotterdam, A.A. Balkema, 3, 2245-2250.

Arreygue-Rocha, E., Hernández-Madrigal, V.M., Garduño-Monrroy, V. H., 2003, Estudio Geotécnico y Geofísico del sector sur (La Mesa) del club campestre, Morelia, Michoacán: Morelia, Universidad Michoacana de San Nicolás de Hidalgo, Instituto de Investigaciones Metalúrgicas, Departamento de Geología y Mineralogía, Reporte, $18 \mathrm{p}$.

Braja, M.D., 1999, Fundamentos de Ingeniería Geotécnica: EUA ,Thomson Learning, $594 \mathrm{p}$.

Crespo-Villalaz; C. 1980, Mecánica de suelos y cimentaciones: México, Limusa, 640p.
Domínguez-Ortíz, F., 2003, Los procesos de remoción en masa y sus relaciones con la sismicidad potencial en el sector de Ocolusen de la falla La Paloma, Morelia, Michoacán; México: Morelia, Universidad Michoacana de San Nicolás de Hidalgo, Facultad de Ingeniería Civil, Tesis de licenciatura, $115 \mathrm{p}$.

Garduño-Monrroy, V. H., Hernández-Madrigal, V.M., Arreygue-Rocha, E., 2002, Estudio Geotécnico y Geofísico del camino en el fraccionamiento Cumbres de las Américas en Ocolusen, Morelia, Michoacán: Morelia, Universidad Michoacana de San Nicolás de Hidalgo, Instituto de Investigaciones Metalúrgicas, Departamento de Geología y Mineralogía, Reporte, 25p.

Garduño-Monrroy, V. H., Arreygue-Rocha, E., Israde-Alcántara, I., Rodríguez-Torres, G., 2001, Efectos de las fallas asociadas a sobreexplotación de acuíferos y la presencia de fallas potencialmente sísmicas en Morelia, Michoacán, México: Revista Mexicana de Ciencias Geológicas, 18 (1), 37-54.

González de Vallejo, L.I., Ferrer, M., Ortuño, L., Oteo, C., 2002, Ingeniería Geológica: Madrid, Pearson Educación, 715p.

Sharma, S., 1991, An Integral slope Stability Analysis, Program for Personal Computers, Interactive software Designs, Inc., Reference Manual, $131 \mathrm{p}$.

Manuscrito recibido: Agosto 4, 2006

Manuscrito corregido recibido: Octubre 27, 2006

Manuscrito aceptado: Diciembre 4, 2006 